\title{
CALIDAD DE VIDA Y SU ASOCIACION CON LA NEGLIGENCIA EN EL CUIDADO DENTAL EN PREESCOLARES
}

\author{
QUALITY OF LIFE AND ITS ASSOCIATION WITH NEGLIGENCE IN DENTAL CARE IN \\ PRESCHOOLS
}

Yvette Giannina Tello-Guerrero giantgold@hotmail.com

ORCID: 0000-0003-1294-0481

\author{
Guido Perona-Miguel de Priego ${ }^{1}$ \\ guidoperona54@gmail.com \\ ORCID: 0000-0003-4092-7364
}

\section{RESUMEN}

Objetivo: Se buscó determinar la frecuencia de los indicadores de la negligencia en el cuidado dental y su asociación con la calidad de vida relacionada a la salud bucal en preescolares de 36 a 71 meses, del Hospital Central de la Fuerza Aérea del Perú (Lima, Perú), en 2016. Metodología: Estudio prospectivo, transversal, descriptivo y observacional en el que se examinó la severidad de la caries de infancia temprana, empleando el sistema ICDAS II; se determinó la presencia de indicadores de la negligencia en el cuidado dental y se utilizó el cuestionario ECOHIS en 187 niños y sus madres. Se utilizaron las pruebas de chi cuadrado, correlación de spearman y regresión logística para determinar la asociación entre caries, calidad de vida y negligencia en el cuidado dental. Resultados: La caries dental tuvo un mayor impacto en la calidad de vida en la dimensión familia. Se determinó una correlación positiva entre caries dental $(p<0,0001)$, educación de la madre en prevención $(p<0,05)$ y calidad de vida.
Seis indicadores de la negligencia en el cuidado dental fueron estadísticamente significativos $(p<0,05)$. Sin embargo, no pudieron ser considerados factores de riesgo para la calidad de vida. Conclusiones: La caries dental tuvo un impacto negativo en la calidad de vida; la educación a la madre en prevención tuvo un impacto positivo en la salud oral y no se puede afirmar que los indicadores de negligencia actúen como factores de riesgo para una mala calidad de vida en los preescolares de este estudio.

Palabras clave: negligencia en el cuidado dental, ECOHIS, calidad de vida

\section{ABSTRACT}

Objective: The aim of this study was to determine the prevalence of oral indicators of dental neglect, and their association with oral health related to quality of life (OHRQoL) in children treated at the Hospital Central de la Fuerza Aérea del Perú in 2016, Lima, Perú. Methods: A cross sectional study was performed in

Citar como: Tello-Guerrero YG, Perona-Miguel de Priego GA. Calidad de vida y su asociación con la negligencia en el cuidado dental en preescolares. Rev Cient Odontol (Lima). 2019; 7 (1): 89-102. 
187 children aged 36-71 months and their mothers. Statistical analysis was performed using the Chi squared test, correlation of Spearman and logistic regression to determine possible associations with early childhood caries, OHRQoL and dental neglect. Results: Tooth decay showed a high negative impact on OHRQoL in the domain of family impact. There was a positive correlation between OHRQoL and tooth decay ( $p<0.0001)$ and the mother's education in aspects related to oral prevention $(p<0.05)$. Six oral indicators of dental neglect were statistically significant $(p<0.05)$, although they could not be considered as risk factors for OHRQoL. Conclusions: Tooth decay has a negative impact on OHRQoL. While we found a positive impact in relation to the mother having received education on oral prevention, indicators of negligence were not demonstrated to be risk factors for OHRQoL.

Keywords: dental neglect, ECOHIS, oral health related, quality of life

\section{INTRODUCCIÓN}

El estado de salud bucal permite determinar el nivel de la calidad de vida relacionada a la salud bucal (CVRSB) en un paciente pediátrico, debido a que las patologías de la cavidad oral sin tratamiento (caries de infancia temprana, enfermedad periodontal, pérdida prematura de piezas dentales o maloclusiones) ocasionan dolor, infección, alteraciones funcionales y estéticas, lo que a su vez tiene un impacto negativo en el crecimiento, desarrollo y bienestar del niño, e incluso afecta el entorno familiar $\left({ }^{1-7}\right)$.

El maltrato infantil por negligencia es la falla, reciente o no, del padre o tutor en cubrir de forma apropiada las necesidades básicas del niño y abarca los aspectos educativos, psicológicos, físicos, emocionales y de salud, ante la cual existe un marco legal en todos los países destinado a proteger a la población infantil $\left({ }^{7-10}\right)$.

Sin embargo, la negligencia en el cuidado dental es un término desconocido en el Perú y ha sido definida como una falla en proveer cuidados odontológicos necesarios para garantizar un buen estado de salud bucal libre de dolor e infección. Esta falta de cuidados puede afectar seriamente la salud y el desarrollo del niño cuando es expuesto a esquemas repetidos de antibióticos por infecciones odontogénicas crónicas 0 severas. Muchos de ellos requieren hospitalizaciones, tratamiento odontológico en sala de operaciones bajo anestesia general, atenciones por urgencias odontológicas repetidas veces, ausencia a sus citas dentales, desnutrición, bajo rendimiento $y$ ausencias escolares $\left({ }^{6-14}\right)$.

Puede presentarse de forma aislada sin afectar algún otro aspecto general del niño; sin embargo, su presencia es un signo de alerta, pues es considerada un indicador odontológico de sospecha de maltrato infantil, debido a que las lesiones dentales sin tratar están a menudo presentes en niveles altos en niños que padecen abuso y abandono, a diferencia del resto de la población infantil $\left({ }^{7}\right)$.

Fisher-Owens, Lukefahr y Tate $\left(^{7}\right)$ elaboraron una guía, en conjunto con la American Academy of Pediatric Dentistry (AAPD) y la American Academy of Pediatrics (AAP), con la finalidad de enfocar el rol que asumen los odontopediatras y pediatras ante los signos clínicos en la región facial y oral de abuso físico, abuso sexual, bullying y maltrato por negligencia, debido al impacto negativo a largo plazo en la salud y el desarrollo físico, emocional, cognitivo, social y emocional del niño. Esto permitió al equipo de salud identificar casos o reportar sospechas. Según el marco 
legal donde desarrolle sus labores y dependiendo de la magnitud del daño ocasionado por la CIT que presente el niño en la cavidad oral, puede requerir solamente un manejo odontológico, una atención integral con otras especialidades de salud o, inclusive, ser referido a un programa de protección al menor cuando estas lesiones coexisten con otros signos clínicos que harían sospechar de un caso de maltrato infantil o abuso, especialmente en una población infantil vulnerable y con alto riesgo social.

En países como EE. UU., Canadá y Reino Unido, la capacitación para todo profesional que trabaja con población infantil es obligatoria con la finalidad de identificar los signos de maltrato y erradicar el temor de reportar alguna sospecha a los servicios sociales $\left(^{8-10,15-26}\right)$.

Bathia y Cols $\left({ }^{22}\right)$ mencionan dos aspectos importantes: la negligencia en el cuidado dental debe diferenciarse de la carencia de recursos económicos para buscar atención dental en una familia y solo podría considerase negligencia cuando existan recursos sanitarios y no sean utilizados, y en segundo lugar que el odontólogo, a pesar de hallarse en una posición estratégica que le permite observar signos de negligencia en el cuidado dental, no la reporte, por desconocimiento o temor.

Se menciona la interacción de varios factores que afectan el entorno familiar y que podrían influir en la ocurrencia de la negligencia en el cuidado dental: la mala salud de los padres, el abuso doméstico, el desempleo, la pobreza, la inmadurez de los padres, el desconocimiento de las necesidades infantiles, un gran número de hijos, enfermedades sistémicas 0 genéticas presentes, el acceso a centros de atención dental y la necesidad de cuidados especiales y altos niveles de estrés, donde la CIT es a menudo un indicador de negligencia en el cuidado dental en los niños provenientes de estos hogares necesitándose una evaluación de los contextos clínicos y sociales en cada caso en particular (8,27-32).

Se considera que es maltrato infantil cuando están presentes los siguientes criterios: presencia de enfermedad dental obvia sin tratamiento, la enfermedad es visible y obvia para una persona sin formación odontológica o de salud, cuando ocasiona un impacto negativo en el niño y se aprecia la falla en la obtención de cuidado odontológico, no limitándose a un conteo de lesiones de caries de infancia temprana $\left({ }^{27,33-37}\right)$.

Baptista y cols. ${ }^{36}$ ) hicieron una revisión sistematizada sobre la negligencia en el cuidado dental con la finalidad de identificar evidencia clínica que permita al odontólogo sospechar su presencia encontrando que los indicadores pueden agruparse en tres grupos: las manifestaciones orales e historia clínica del niño, los determinantes sociales del niño y las características de los padres, y encontraron que la CIT, el dolor orofacial, la presencia de más de dos tercios de placa en la superficie dental, el sangrado gingival, la demora en la búsqueda de atención dental, la falla para cumplir las indicaciones de tratamiento y permitir el deterioro de la salud oral del niño son características reconocibles de negligencia en el cuidado dental.

No existen estudios en Perú en los que se mencione la negligencia en el cuidado dental y la presente investigación buscó determinar la asociación entre la calidad de vida relacionada a la salud bucal de una población preescolar y los indicadores clínicos presentes en la negligencia en el cuidado dental, con la finalidad de comprender la dimensión en que esta entidad es capaz de afectar de manera negativa la salud bucal de la población pediátrica. Asimismo, en vista que el término negligencia en el cuidado dental 
es desconocido y no es aplicado en el Perú, se propone incluirlo como término odontológico en las documentos normativos y leyes con la finalidad de proteger a la población infantil $\left({ }^{38-40}\right)$.

\section{MATERIALES Y MÉTODOS}

Este estudio observacional y transversal contó con una muestra de 178 niños de 36 a 60 meses y sus respectivas madres quienes acudieron a la sección de Odontopediatría del Departamento de Estomatología del HCFAP de la ciudad de Lima durante en 2016.

Los criterios de selección fueron: pacientes pediátricos de ambos sexos de edades comprendidas entre los 36 y 60 meses, en aparente buen estado de salud general, que presenten dentición primaria completa y que acudan a la consulta acompañados por sus madres y los criterios de exclusión fueron: pacientes cuyas madres no deseaban participar en la investigación, presencia de enfermedades sistémicas o alguna discapacidad física o mental y pacientes que no presenten piezas dentarias primarias completas.

El estudio fue revisado y aprobado por el Comité de Ética de la Universidad Científica del Sur y se contó con la autorización $y$ firma del consentimiento informado por parte de la madre antes de proceder a realizar las pregunta del cuestionario y examinar a los niños.

Se realizó la calibración previa al estudio teórica, practica y clínica a cargo de un Gold Standard cuyo resultado de calibración fue de 0,84 intraexaminador y 0,81 interexaminador. Asimismo, se llevó a cabo un estudio piloto para obtener el tamaño muestral a través de una fórmula muestral para estimar una proporción con un nivel de confianza del $95 \%$, una precisión del $3 \%$ y comprobar la precisión de la ficha de recolección de datos validada por un juicio de expertos.

Se empleó un cuestionario estructurado donde se obtuvo la información sociodemográfica del paciente $y$, posteriormente, fue examinando para registrar la presencia de las lesiones de caries dental según el método ICDAS II por pieza dentaria empleando una sonda OMS. Posteriormente, se determinó la asociación entre las lesiones de caries y el impacto en la calidad de vida, según el cuestionario ECOHIS (Early Childhood Oral Health Impact Scale), que es un instrumento que consta de 13 ítems los cuales evalúan la percepción del padre con respecto a las limitaciones que ha tenido el niño por presentar patologías orales y el impacto de estas en la calidad de vida de su hijo y en la familia. El cuestionario fue respondido usando la escala de Likert, que consta de respuestas que van desde "nunca" a "muy a menudo" (equivalente a una puntuación de 0 a 4 , respectivamente).

Por último, fueron registrados otros hallazgos clínicos intraorales mencionados en la literatura científica como indicadores de negligencia en el cuidado dental agrupados de acuerdo con las sugerencias de los expertos consultados.

Los datos fueron analizados mediante hojas de cálculo Excel y el programa Stata 14 para Windows. Se utilizaron la estadística descriptiva para las variables cualitativas, la prueba chi cuadrado para evaluar la asociación entre las variables Indicadores de la negligencia en el cuidado dental y calidad de vida con un valor $p<0,005$ para establecer la asociación estadísticamente significativa.

Asimismo, se empleó la correlación de Spearman y la regresión logística para evaluar cómo las covariables influyen en la asociación entre los indicadores de la negligencia en el cuidado dental y la 
calidad de vida. Se utilizaron gráficos de cajas y bigotes para las variables calidad de vida y estado de salud bucal.

Se realizó una adaptación transcultural del término dental neglect a negligencia en el cuidado dental, en vista que la traducción: negligencia dental es similar al término negligencia odontológica, el cual se refiere a la mala praxis dental en el Perú.

\section{RESULTADOS}

Se observó una mayor frecuencia de pacientes del sexo femenino y un mayor número de pacientes examinados correspondieron al grupo de $>60$ meses de edad (tabla 1).

Se halló que la prevalencia de pacientes libres de caries fue del $30 \%$, mientras que el $70 \%$ presentaron lesiones de CIT en diversos estadios según la severidad, siendo la caries severa la más frecuente, con un $38 \%$ (tabla 2 ).

Seencontró, segúnlaescalaECOHIS, que el mayor impacto de la CIT correspondió a la dimensión impacto en la familia (m = 4), mientras que la dimensión Impacto en el niño $(m=1)$ obtuvo un valor menor (tabla 3 y gráficos 1 y 2 ).

La correlación entre la CIT y la CVRSB fue significativa: $p=0,0001(p<0,001)$ (tabla 4) y se observó una asociación significativa entre la CIT y la instrucción de la madre en cuidados preventivos odontológicos en sus hijos (tabla 5).

La CIT $(70 \%)$, la presencia de placa $(62,6 \%)$ y la gingivitis asociada a placa $(51,3 \%)$ tuvieron una mayor prevalencia en los niños examinados y se encontró una asociación significativa $(p<0,05)$ entre la presencia de seis (06) indicadores de la negligencia en el cuidado dental con la CVRSB, debido al impacto negativo que ocasionaron en el paciente: "Presenta dolor", "Primera vez que acude al Odontopediatra", "Registra ausencia a citas/controles", "Presenta placa bacteriana", "Presenta gingivitis asociada a placa," " Presenta CIT", "Presenta fistulas/ abscesos" y "Presenta +3 lesiones de caries activas" (tabla 6).

Tabla 1. Distribución de los pacientes según la edAd y El SeXo. Hospital faP 2016

\begin{tabular}{|l|c|c|c|c|c|c|c|c|}
\hline Sexo & \multicolumn{9}{|c|}{ EDAD (meses) } \\
\hline Femenino & $36-47$ & $\%$ & $48-59$ & $\%$ & $60-71$ & $\%$ & Total & $\%$ \\
\hline Masculino & 25 & 13,4 & 29 & 15,5 & 44 & 23,5 & 98 & 52,4 \\
\hline Total & 21 & 11,2 & 26 & 13,9 & 42 & 22,5 & 89 & 47,6 \\
\hline
\end{tabular}

Fuente: Elaboración propia, ficha de recolección de datos

Tabla 2. Frecuencia de la severidad de las lesiones de Caries de Infancia Temprana en PREESCOLARES EMPLEANDO EL SISTEMA ICDAS II. Hospital FAP 2016

\begin{tabular}{|l|c|c|}
\hline Estadio & N. ${ }^{\circ}$ pacientes examinados & $\%$ \\
\hline Sin lesión & 57 & 30 \\
\hline Caries inicial ( 1 y 2) & 27 & 14 \\
\hline Caries establecida (3 y 4) & 33 & 18 \\
\hline Caries severa ( 5 y 6 ) & 70 & 38 \\
\hline Total & 187 & 100 \\
\hline
\end{tabular}

Fuente: Elaboración propia, ficha de recolección de datos 
Tabla 3. Medidas de tendencia central de los puntajes del ECOHIS Según Impacto en EL NiÑo Y EN LA FAMILIA. HOSPITAL FAP 2016

\begin{tabular}{lcccccc}
$\begin{array}{l}\text { CVRSB } \\
\text { ECOHIS }\end{array}$ & $\begin{array}{c}\text { Número de } \\
\text { preguntas }\end{array}$ & Media & $\begin{array}{c}\text { Desviación } \\
\text { estándar }\end{array}$ & Mediana & $\begin{array}{c}\text { Desviación } \\
\text { intercuartil }\end{array}$ & $\begin{array}{c}\text { Rango } \\
\text { observado }\end{array}$ \\
Impacto en el niño & 1 & 0,7 & 0,9 & 0 & 0,5 & $0-4$ \\
$\begin{array}{l}\text { Síntomas orales } \\
\text { Limitaciones funcionales }\end{array}$ & 4 & 1 & 1,6 & 0 & 1 & $0-9$ \\
\hline $\begin{array}{l}\text { Aspectos psicológicos } \\
\text { Autoimagen/interacción }\end{array}$ & 2 & 1,1 & 1,3 & 1 & 1 & $0-5$ \\
social & 2 & 0,3 & 0,7 & 0 & 0 & $0-4$ \\
Total & 9 & 3,1 & 4,5 & 1 & 2,5 & $0-18$ \\
Impacto en la familia & & & & & & 0 \\
Angustia de los padres & 2 & 2,5 & 2,1 & 2 & 2 & $0-8$ \\
\hline $\begin{array}{l}\text { Función familiar } \\
\text { Total }\end{array}$ & 2 & 1,8 & 1,7 & 2 & 1,5 & $0-8$ \\
$\begin{array}{l}\text { Puntuación total } \\
\text { ECOHIS }\end{array}$ & 4 & 4,4 & 3,8 & 4 & 3,5 & $0-15$ \\
\hline
\end{tabular}

Fuente: Elaboración propia, ficha de recolección de datos

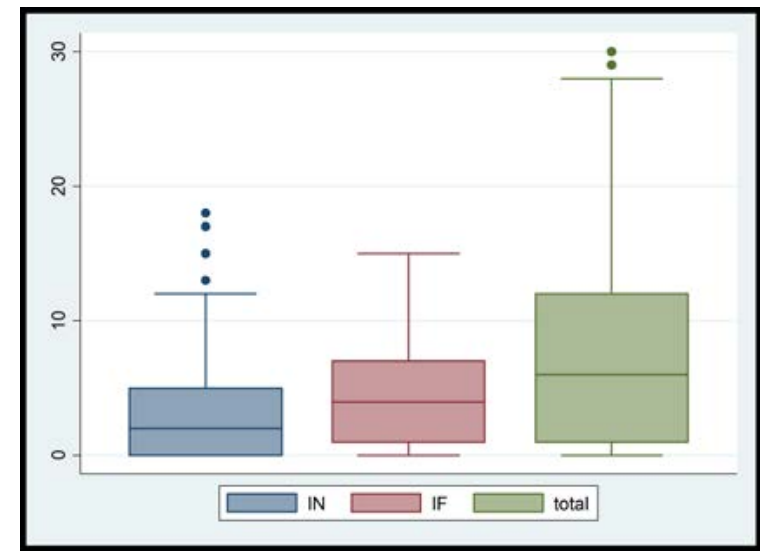

GrÁFICO 1. Distribución dE LOS PUNTAJES DEL ECOHIS SEGÚN IMPACTO EN EL NIÑO E IMPACTO EN LA FAMILIA. HOSPITAL FAP 2016

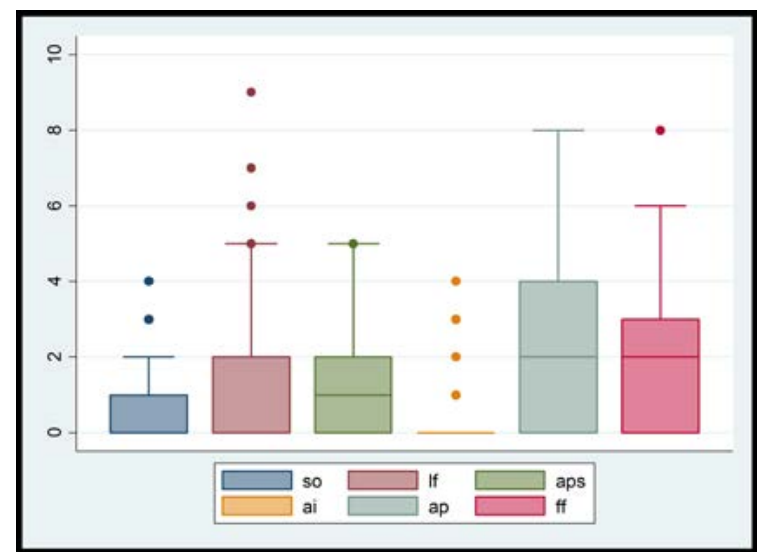

Gráfico 2. Distribución de LOS PUNTAJes del ECOHIS según LAS dimensiones. HospitAl FAP 2016 
TABLA 4. CorRelación ENTRE CARIES de INFANCIA TEMPRANA Y LA CALIDAD DE VIDA RELACIONADA A LA SALUD BUCAL. HOSPITAL FAP 2016



*Prueba de Correlación de Spearman.

Fuente: Elaboración propia.

TABLA 5. Asociación entre LA CARIES DE INFANCIA temprana Y LA INSTRUCCIÓN DE LA MADRE EN CUIDADOS PREVENTIVOS. HOSPITAL FAP 2016

\begin{tabular}{|c|c|c|c|c|c|c|c|}
\hline \multirow{3}{*}{$\begin{array}{l}\text { Caries de infancia } \\
\text { temprana } \\
\text { (n. } .^{\circ} \text { de lesiones) }\end{array}$} & \multicolumn{4}{|c|}{$\begin{array}{l}\text { Madre recibió instrucción de cuidados } \\
\text { preventivos en clínica de bebés }\end{array}$} & \multirow{2}{*}{\multicolumn{2}{|c|}{ Total }} & \multirow{3}{*}{$\begin{array}{c}\mathrm{Chi}^{2} \\
\text { (valor de } \mathrm{p} \text { ) }\end{array}$} \\
\hline & \multicolumn{2}{|c|}{ NO } & \multicolumn{2}{|c|}{ SI } & & & \\
\hline & $\mathrm{N}$ & $\%$ & $\mathrm{~N}$ & $\%$ & $\mathrm{~N}$ & $\%$ & \\
\hline Sanos & 9 & 4,81 & 48 & 25,67 & 57 & 30,48 & $0,027^{*}$ \\
\hline 1 a 3 caries & 17 & 9,09 & 32 & 16,58 & 48 & 25,67 & ------ \\
\hline 4 a 6 caries & 19 & 10,16 & 28 & 14,97 & 47 & 25,13 & ----- \\
\hline$>7$ caries & 13 & 6,95 & 22 & 11,76 & 35 & 18,72 & ------ \\
\hline Total & 58 & 31,02 & 129 & 68,98 & 187 & 100 & ----- \\
\hline
\end{tabular}

Fuente: Elaboración propia $p<0,05$

TABLA 6. FreCUENCIA dE INDICADORES CLíNICOS DE LA NEGLIGENCIA EN EL CUIDADO DENTAL EN PREESCOLARES Y SU ASOCIACIÓN CON LA MALA CALIDAD DE VIDA RELACIONADA A LA SALUD BUCAL. HOSPITAL FAP 2016

\begin{tabular}{|l|c|c|c|c|c|c|c|c|}
\hline $\begin{array}{l}\text { Indicadores de la negligencia e el } \\
\text { cuidado dental }\end{array}$ & \multicolumn{2}{|c|}{ Sí } & \multicolumn{2}{|c|}{ No } & \multicolumn{2}{c|}{ Total } & \multicolumn{2}{c|}{ Chi 2} \\
(Valor de p)
\end{tabular}

Fuente: Elaboración propia

$p<0,01$ 
TABLA 7. ANÁLISIS MULTIVARIAdO DE REGRESIÓN LOGíSTICA PARA EVALUAR LA INFLUENCIA DE LOS INDICADORES DE LA NEGLIGENCIA EN EL CUIDADO DENTAL SOBRE LA MALA CALIDAD DE VIDA RELACIONADA A LA SALUD BUCAL. HOSPITAL FAP 2016

\begin{tabular}{|c|c|c|c|c|c|c|}
\hline $\begin{array}{l}\text { Indicadores de la negligencia en el } \\
\text { cuidado dental }\end{array}$ & Coef. & Std. Err & z & $P>|z|$ & \multicolumn{2}{|c|}{ IC $95 \%$} \\
\hline 1. Presenta dolor & 0 & (omitido) & & & & \\
\hline $\begin{array}{l}\text { 2. Primera vez que acude al odontope- } \\
\text { diatra }\end{array}$ & 1318 & 6767007 & 0,19 & 0,846 & $-1,194$ & 1,458 \\
\hline 3. Registra ausencia a citas/ controles & 1,445823 & 8039793 & 1,80 & 0,072 &,- 129 & 3,021 \\
\hline 4. Presencia de placa bacteriana &,- 2823338 & ,5332692 & $-0,53$ & 0,597 & $-1,327$ & ,762 \\
\hline 5. Presenta gingivitisasociada a placa & $\begin{array}{c}1,4 \\
21402\end{array}$ & 6566481 & 2,16 & $0,030^{*}$ & 134 & 2,708 \\
\hline 6. Presenta CIT & ,4197965 &, 5402331 & 0,78 & 0,437 &,- 639 & 1,478 \\
\hline 7. Presenta fistulas /abscesos & 0 & (omitido) & & & & \\
\hline 8. Presenta +3 caries activas & ,9348483 & 7098676 & 1,32 & 0,188 &,- 456 & 2,326 \\
\hline
\end{tabular}

Fuente: Elaboración propia

Sin embargo, el análisis multivariado de regresión logística mostró en este modelo logístico el indicador "Gingivitis asociada a placa" ( $p<0,05)$, que a pesar de haber sido el único indicador con una valor significativo, no puede ser considerado un factor de riesgo para la variable respuesta CVRSB ( $p=0,03$; IC 95\%: 0,134 a 2,708) (tabla 7).

No se encontró ninguna asociación entre las variables edad de la madre, grado de instrucción, estado civil, persona responsable de cuidar el niño en casa con CIT y CVRSB ( $p>0,05)$.

\section{DISCUSIÓN}

La CIT genera un impacto negativo en el estado de salud bucal y general del niño y afecta de forma significativa al entorno familiar $\left({ }^{12-14,16,18,35,36}\right)$, como se observó en los resultados de esta investigación, en la que el $70 \%$ (130 niños) presentaron lesiones de caries en diversos estadios según el sistema ICDAS II, siendo este valor mayor que lo reportado por Torres y cols. $\left({ }^{11}\right)$ sobre frecuencia de caries en niños provenientes de áreas urbano-marginales (62,3\%).
La población estaba conformada por preescolares de 60 a 71 meses $(45,9 \%)$, de procedencia urbana, cautiva, con acceso a un sistema de salud propio y asegurada en un centro hospitalario donde por primera vez se aplicó el sistema ICDAS II.

Los resultados de la escala ECOHIS mostraron que el mayor impacto fue en la dimensión familia $(m=4)$, lo que no coincide con lo reportado por la literatura, la cual señala que las dimensiones más afectadas están relacionadas con el niño $\left(^{3,4,1}\right)$. Es posible que este resultado se deba al tipo de trabajo altamente demandante que uno o ambos padres realizan, donde la dinámica familiar fue desestabilizada en mayor grado a causa de la caries dental.

Se encontró una correlación significativa entre el impacto de la CIT en la CVRSB ( $p<0,001)$, que coincide con numerosos autores $(2,3,4,5,11)$ y refuerza el concepto de que la prevención y el tratamiento de las patologías bucales evitan el impacto negativo en la CVRSB en la salud y bienestar del niño, mientras que la educación sobre prevención dental en la madre mostró el impacto que tiene en la salud de sus hijos $(p<0,05)$. 
No existen investigaciones en el Perú sobre el impacto que la negligencia en el cuidado dental ocasiona en la calidad de vida del niño, por lo que se buscó, en una primera fase, determinar la asociación entre los hallazgos clínicos intraorales en pacientes preescolares y la CVRSB. Dichos hallazgos son los indicadores odontológicos mencionados en la literatura y presentes en los pacientes examinados. Fue notorio observar el impacto significativo que ocasionaron en la calidad de vida del niño tal y como se observó en este estudio.

Bathia y cols. ${ }^{22}$ ) afirman que la negligencia en el cuidado dental es una entidad multifactorial y multidimensional, por lo que deben de considerarse muchos factores de riesgo presentes que están relacionados con los padres, el entorno social y cultural, o el niño, mientras que Haugseth $\left({ }^{34}\right)$ y Harris y Wittington ${ }^{(35}$ ) hacen énfasis en que la negligencia en el cuidado dental es evaluada en tres aspectos: la presencia de la enfermedad de manera obvia, el impacto significativo en su salud y la falla para obtener un cuidado apropiado, y que no se basa tan solo en un número de caries presentes, sino que se trata del análisis de la magnitud del impacto asociado con otros factores presentes.

Es notorio observar que seis indicadores de la negligencia en el cuidado dental: "Presencia de dolor", "Registra ausencia a citas", "Presenta placa bacteriana", "Presencia de Gingivitis asociada a placa", "Presenta CIT" y "Presenta +3 lesiones de caries activas" resultaron significativamente estadísticos al ser asociados con la variable CVRSB ( $p<$ 0,05 ) (tabla 6); sin embargo, al determinar la fuerza de la asociación, se encontró que el único indicador que resultó estadísticamente significativo $(p=0,030)$ fue la "Gingivitis asociada a placa", que en este modelo logístico no resultó ser un factor de riesgo para la CVRSB (IC 95\%:
0,134 a 2,708) (tabla 7). Este hallazgo es peculiar debido a que la presencia de placa bacteriana de manera constante favorece el desarrollo de la gingivitis y aumenta el riesgo de desarrollar CIT; no se determinó que sea un factor de riesgo en este estudio.

La presencia de la gingivitis asociada a placa bacteriana es un indicador clínico que refleja una inadecuada remoción mecánica de la placa bacteriana por los padres o cuidadores en un preescolar carente de la motricidad apropiada para una correcta higiene. Haugsseth34 afirma que la negligencia en el cuidado dental es a menudo un componente de la negligencia general y, cuando es detectada, los profesionales de la salud deben considerar y buscar la presencia de negligencia en el cuidado dental que incluyen entre otras características una higiene oral deficiente. En este estudio pudo ser posible que la influencia de otras variables haya determinado el resultado obtenido.

En el presente estudio, se registraron signos clínicos sugerentes de negligencia en el cuidado dental y que si bien es cierto destacan la falla o la demora en la búsqueda de la atención odontológica por parte de las madres, no se puede considerar únicamente el registro del número de lesiones de caries dental presentes, sino también la presencia de enfermedad gingival, dolor o infecciones crónicas sin resolver, y el impacto significativo en el niño relacionado con otras características asociadas a los determinantes sociales de la familia y la evaluación psicosocial de la familia $\left({ }^{22,35,36}\right)$, las cuales no fueron analizadas con detenimiento en esta investigación por no formar parte de sus objetivos, pero que probablemente sí pudieron haber influido de manera negativa en el estado de salud del niño. 
Existen estudios en los que se menciona que los niños afectados por la dinámica de un hogar de condiciones sociales bajas donde coexisten problemas domésticos de negligencia, abuso o uso de sustancias o alcohol, presentaron altos niveles de caries, placa bacteriana, sangrado gingival y una falla en la continuidad de las citas y ausencia a las mismas en un periodo de dos años $\left({ }^{22,36}\right)$, en comparación con grupos controles.

En esta investigación, se encontró que los dos casos con mayor impacto negativo en la CVRSB pertenecieron a dos pacientes cuyas madres se encontraron afrontando un proceso de divorcio y de tenencia de la patria potestad solicitada por el otro cónyuge por maltrato por negligencia.

El rol del odontólogo en la sociedad contempla también el peritaje legal y la valoración del daño en casos de abuso físico, como parte del equipo forense, ante lo cual es necesario vislumbrar que, en un futuro, la labor del odontopediatra en el Perú necesariamente incorporará la misión de proteger a la población infantil en riesgo $\left({ }^{41}\right)$, como es usual en otros países, en donde las leyes y sociedades científicas pediátricas consideran una obligación legal y ética el reportar los casos de sospecha de abuso y de negligencia en el cuidado dental (7-10,20,23). Por ello, su labor y experiencia clínica deben ser tomadas en cuenta por la ley para la toma de decisiones respecto de la situación legal de un menor de edad y para determinar el interés superior del niño cuando su salud, vulnerabilidad y seguridad se encuentren comprometidos $\left({ }^{38}\right)$, como sucede en los casos en los que se justifique una acción legal de tenencia infantil. Por esta razón, se debe considerar la capacitación en la especialidad de odontología pediátrica, con la finalidad de conducirse de manera eficiente y profesional en los casos de peritajes legales requeridos por la ley, a fin de completar el enfoque global de protección ausente en la actualidad de la labor del odontopediatra.

\section{CONCLUSIONES}

Existe una asociación significativa entre la CIT y la CVRSB ( $p<0,001$ ) y la instrucción de la madre respecto a cuidados preventivos y CIT $(p<0,05)$ en los preescolares evaluados en la Sección de Odontopediatría del Hospital FAP, en 2016.

Seis (06) indicadores de negligencia en el cuidado dental mostraron una asociación estadísticamente significativa con la CVRSB; sin embargo, a pesar de los resultados, no se puede afirmar que la gingivitis asociada a placa $(p=0,030)$ sea un factor de riesgo para la CVRSB en este modelo de estudio (IC 95\%: 0,134 a 2,708), al igual que en el caso los otros indicadores.

Contribución del autor: Yvette Giannina Tello Guerrero y Guido Perona-Miguel de Priego han participado en la concepción del artículo, la recolección de información, su redacción y la aprobación de la versión final.

Fuente de financiamiento: Autofinanciado.

Conflicto de interés: Los autores declaran no tener conflicto de interés de ningún tipo. 


\section{REFERENCIAS BIBLIOGRÁFICAS}

1. Albites U. Validación de tres instrumentos para medir la calidad de vida relacionada a la salud bucal en niños peruanos de 11 a 14 años de edad. Lima 2011. [Tesis de Especialidad]. Lima: Universidad Científica del Sur; 2013.

2. Sherham A, Tsakos G, Abanto J, Bonecker M. Epidemiología de la caries dental e impacto en la calidad de vida. En: Bonecker M, Abanto J, Salete N, Petorosi J, Guedes Pinto A. (editores). Problemas bucales en odontopediatría. Madrid: Ripano; 2014.

3. López P, García C, Villena R, Bordoni N. Cross cultural adaptation and validation of the early childhood health impact scale (ECOHIS) in children preschooler. Acta Odontol Latinoam. 2013. 26 (2): 60-7.

4. López P, García C. Calidad de vida y problemas bucales en preescolares de la provincia de Huaura, Lima. Rev. Estomatol Hered. 2013. 23 (3): 139-47.

5. Bonecker M, Abanto J, Tello G, Butini L. Impact of dental caries on preschool children's quality of life: an update. Braz Oral Res. 2012; 26 (1): 103-7.

6. Yawary R, Anthonappa R, Eckambaran M, Mc Grath C, King N. Changes in the oral health related quality of life in children following comprehensive oral rehabilitation under general anaesthesia. Int J. Paediatr Dent. 2015. [Epub ahead of print]. Disponible en http://onlinelibrary.wiley.com/doi/10.1111/ipd.12200/epdf?r3 referer=wol\&tracking_action=preview_click\&show_checkout=1\&purchase _ referrer=www.ncbi.nlm.nih.gov

7. Fischer-Owens SA, Lukefahr JL, Tate AR, AAP American Academy of Pediatrics, Section Oral Health Committee on child abuse and neglect, American Academy of Pediatric Dentistry, council of clinical affairs, council on scientific affairs, ad hoc work group on child abuse and neglect. Oral and Dental Aspects of Child Abuse and Neglect. Pediatrics. 2017; 140 (2): e20171487.

8. Harris J, Balme RC, Sidebotham P. British Society of Paediatric Dentistry: a policy document on dental neglect in children. Int J Paediatr Dent. 2009; 1-8

9. Markovic N, Arslanagic A, Kovaslija S, Bajric E, Selimovic-Dragas M et al. Knowledge and attitudes regarding child abuse and neglect. Mater Sociomed. 2015; 27 (6): 372-5.

10. Bradbury C, Innes N, Evans D, Ballantyne F, Taylor J. Dental neglect as a marker of broader neglect: a qualitative investigation of public health nurses' assessments of oral health in preschool children. BMC Public Health 2013; (13): 1370-82.

11. Torres G, Blanco D, Chávez M, Apaza S, Antezana V. Impacto de la caries de la infancia temprana en la calidad de vida relacionada a la salud bucal en niños peruanos menores de 71 meses de edad. Odontol Sanmarquina 2015; 18 (2): 87-94.

12. Sheiman A. Dental caries affects body weight, growth and quality of life in preschool children. British Dent Journal 2006; 201 (10): 625-6. 
13. Schuch H, Costa D, Torriani D, De Marco F, Goettems M. Oral Health related of schoolchildren: impact of clinical and psychosocial variables. Int J Paediatr Dent. 2015; 25 (5): 358-65.

14. Arrow P, Raheb J, Miller M. Brief Oral health promotion intervention among parents of young children to reduce early childhood dental decay. BMC Public Health 2013; (13): 245-53.

15. Valencia N, Lawrence H, Goodman D. Prevalence of early caries in a population of children with history of maltreatment. Journal of Public Health Dent. 2008; G8 (2): 94-101.

16. Monse B, Heinrich-Weltzien R, Benzian $\mathrm{H}$, Holmgren $\mathrm{C}$, Van Palestein Helderman w. PUFA. An index of clinical consequences of untreated dental caries. Community Dent Oral Epidemiol. 2010; 38: 77-82.

17. American Academy Of Pediatric Dentistry. Policy on Early Childhood Caries (ECC): Classifications, consequences and preventive strategies. 2014; 37 (6) 50-2.

18. American Academy Of Pediatric Dentistry. Policy on Early Childhood Caries (ECC): Unique challenges and treatment options. 2014; 37 (6): 53-5.

19. Hibbard R, Sanders B. Abuso infantil y negligencia. En: Dean J, Avery D, Mc Donald R. Odontología para el niño y el adolescente de Mc Donald y Avery. $9^{\circ}$ ed. Venezuela: Amolca; 2014.

20. Nuzzolese $E$ et al. Child abuse and dental neglect: the dental team's rol in identification and prevention. Int J Dent Hygiene 2009; 7 (2): 96-101.

21. Lourenco CB, Saintrain MV, Vieira AP, Child, neglect and oral health. BMC Pediatrics. 2013; 13: 188.

22. Bathia $S$ et al. Characteristics of child dental neglect: a systematic review. J Dent. 2014; 42 (3): 229-39.

23. De Panfilis D. Child Neglect: a guide for prevention, assessment and intervention. U.S. Department of Health and Human services. Administration for Children and Families. Administration on Children, Youth and Families. Children's Bureau, Office on Child Abuse and Neglect. 2006. Disponible en https://www.childwelfare. gov/pubPDFs/neglect.pdf

24. Kumar A, Puranik M, Sowmya K. Dental neglect- a review. RR: J Dent Sciences 2015; 3 (1).

25. Rajeshawari R, Rajender R, Manjula M, Srilakshmi. Dental neglect. Indian J Dent Advancements 2010; 2 (1): 138-41.

26. Harris J, Firth L, Chadwick B. Clinical audit of children's missed dental appointments in a city-wide salaried community dental service in relation to guidance on when to suspect child maltreatment. Child Abuse Rev. 2015; Published online in Wiley Online Library Version of Record online: 16 Nov 2015, DOI: 10.1002/car.2419 Disponible en: http://onlinelibrary.wiley.com/advanced/search/results 
27. Barron C, Jenny C. Definition and categorization of child neglect. En: Barron C, Jenny C. Editors. Child Abuse and Neglect: Diagnosis, Treatment and Evidence. Canadá: Elsevier-Saunders; 2011.

28. Fisterra. Guía Clínica del Maltrato Infantil: Maltrato Infantil: Indicadores físicos y comportamentales en el menor. 2015. Disponible en : http:// fisterra.com/guíasclínicas/indicadores-maltrato-infantil/

29. Al Hasbi S, Roberts G, Attari N, Parekh S. A survey of attitudes, knowledge and practice of dentists in London towards child protection. Are children receiving dental tratment at the Eastman Dental Hospital likely to be on the child protection register? Br Dent J 2009; 206 (4) Published online: 13 February 2009 doi:10.1038/ sj.bdj.2009.113 Disponible en http://www.nature.com/bdj/journal/v206/n4/full/ sj.bdj.2009.113.html

30. Maguire S, Naughton A. Neglect: widespread, damaging and difficult to identify. Pediatrics and Child Health. 2016; 26 (11): 485-7.

31. Harris J, Elcock C, Sidebotham P, Welbury R. Safeguarding children in dentistry: 1. Child protection training, experience and practice of dental professionals with an interest in paediatric dentistry. Br Dent J 2009; 206 (8): 409-14.

32. Harris J, Elcock C, Sidebotham P, Welbury R. Safeguarding children in dentistry: 2. Do paediatric dentists neglect child dental neglect? Br Dent J 2009; 206 (9): 465-70.

33. Pushpalatha C, Tammannavar P, Nimbal A, Jain S. Is Dental Neglect a Child abuse? A review. Int J A Res. 2013; 1 (5): 273-5.

34. Haugseth R. Dental Neglect. In: Barron C, Jenny C. Editors. Child abuse and neglect: diagnosis, treatment and evidence. Canadá: Elsevier-Saunders; 2011.

35. Harris J, Wittington A. Dental neglect in children. Paediatrics and Child Health. 2016; 26 (11): 478-84.

36. Baptista AS, Laranjo E, Norton A, Andrade D, Areias C, Macedo A. Dental Neglect, One type of child abuse- Narrative review. Medical Express. 2017; 4 (3): M170301. Disponible en: http://www.scielo.br/scielo.php?script=sci_arttext\&p id=S2358-04292017000300001

37. Keene E, Skelton R, Day P, Munyombwe, Balmer R. The dental health of children subject to a child protection plan. In J of Paed Dent 2015; 25 (6): 428-35.

38. Fernández EW. El derecho del niño a mantener vínculos familiares con ambos progenitores. Revista del Foro 2016; 103: 143-57.

39. Código de las Niñas, Niños y Adolescentes. 2015. Perú. Mimdes. Disponible en: https://www.mimp.gob.pe/files/direcciones/dgnna/Codigo_Ninos_Adolescentes. pdf

40. Colegio Odontológico del Perú. Código de Ética y Deontología. Lima: Esquema; 2016. 
Yvette Giannina Tello-Guerrero, Guido Alberto Perona-Miguel de Priego

41. Naganawa T, Kumar A, Ando T. Recommendations to include dentists/oral surgeons in child abuse prevention teams of general hospitals in Japan. Journal of Oral and Maxillofacial Surgery, Medicine, and Pathology. 2016; 28 (3): 263.

ARtículo RECIBIDO: 12-02-2019

Artículo ACEPTADO: 17-04-2019 